\section{P101 Qualities, Activities and Modes of Operation of Well-Integrated New York City School Gardens}

Kate Gardner, MS, RD, kwg2104@tc.columbia.edu, Teachers College, Columbia University, 525 West 120th Street, Box 137, New York, NY 10025; P. Koch, EdD, RD; I. Contento, PhD

Objective: New York City School Gardens are highly varied in their characteristics, activities, and how they operate within a school. This study aimed to describe the qualities of well-integrated school gardens. That is, those who have a physically maintained garden that students visit regularly and has become a sustained part of the school culture. Design, Setting and Participants: School gardeners from 21 schools in New York City participated in a survey, observation(s), a semi-structured interview, and a concept mapping exercise during the 2013-2014 school year. Photographs were taken of gardens and additional documents were collected for analysis (when available).

Outcome Measures and Analysis: Descriptive statistics were used to analyze the survey and qualitative techniques, including pattern matching, explanation building, and cross-case synthesis were used to analyze the qualitative data. Finally, multidimensional scaling and hierarchical cluster analysis was used to assess and synthesize data collected from the concept mapping exercises.

Results: A rich description, supplemented with images, of each school gardening program was constructed that describe each schools unique coupling of School Garden Integration Framework (SGIF) components to meet their special needs and sustain the garden (e.g. a school with a greenhouse-enclosed rooftop hydrofarm classroom operationalized the SGIF very differently than a school with outdoor raised beds). Taken together these data illustrate the variation in how the SGIF can be used to overcome barriers and effectively integrate a school garden.

Conclusions and Implications: Lessons learned from each school may be helpful for other urban school gardeners and can be used to develop guiding principles and policies for schools with similar gardening needs or requirements.

Funding: None.

\section{P102 Student Perspectives on Nutrition and Health at a Military College}

Deanne Moosman, MS, moosmanDL@vmi.edu, Virginia Military Institute, Dept. of Physical Education, 320 Cocke Hall, Lexington, VA 24473

Objective: The goal of this study was to determine nutritional and health perceptions of students at a military college. The information will be used to develop a larger scale nutritional study aimed at promoting better eating habits in college students.

Design, Setting and Participants: A total of 426 cadets participated in either a web-based or Scantron survey. Information was gathered about demographics, fruit and vegetable consumption, incentives for healthier eating, and attitudes toward nutritional genomics and epigenetics.

Outcome Measures and Analysis: Survey results were assessed using Principal Components Analysis, followed by univariate tests to examine whether attitudes and eating habits varied with demographics or education.

Results: Most cadets (96\%) reported exercising $>1$ hour/ day and a majority (62\%) had a normal BMI (18.5-24.9). Most (95\%) had taken a health course previously and rated their nutritional knowledge as average, above average, or excellent (95\%). But nutrition education or knowledge did not appear to influence fruit or vegetable consumption or attitudes towards nutrition and disease. Cadets reported more interest in changing eating habits for the sake of improving athletic performance (63\%), rather than to avoid disease (8\%).

Conclusions and Implications: Results suggest that, in this population of college students, education alone may not change nutritional habits. Efforts to develop better eating habits should consider motives of the students.

Funding: None.

\section{P103 Food Addiction Among College Students in an Introductory Nutrition Course}

Susan Lewis, BS, susanll@bgsu.edu, Bowling Green State University, 206 Johnston Hall, Bowling Green, OH 43403; M. Anderson, MFN, RD, LD; R. Pobocik, PhD, RD;

M. Ludy, PhD, RD

Objective: College students are vulnerable to weight management problems. This study's objective was to characterize addiction to high-fat, high-sugar foods among students in an introductory nutrition course.

Design, Setting and Participants: Using a crosssectional design, 199 students aged $19.4 \pm 2.7$ years were recruited from their class early in the fall 2014 semester. Students were predominantly female (74.4\%), Caucasian (81.9\%), and underclassmen (22.6\% freshmen, $45.2 \%$ sophomores). The survey included the Yale Food Addiction Scale and demographics. It was introduced in class and self-administered electronically.

Outcome Measures and Analysis: Responses were categorized into symptom groups resembling the criteria for substance dependence outlined in the Diagnostic and Statistical Manual of Mental Disorders. Clinical impairment reflected food behavior that "causes significant distress" and "problems in my ability to function effectively." Food addiction diagnosis required at least three (of seven) symptoms plus clinical significance. Students also identified individual problem foods. Results were characterized with descriptive statistics.

Results: Students exhibited $2.1 \pm 1.6($ mean \pm SD) food addiction symptoms: loss of control (22.6\% of respondents), unsuccessful quitting (92.5\%), time spent to obtain/use/recover (15.6\%), giving up other activities (21.6\%), continued despite problems $(24.6 \%)$, tolerance 